\title{
Ionic Liquids: First Direct Determination of their Cohesive Energy
}

\author{
Luís M. N. B. F. Santos, ${ }^{\dagger}$ José N. Canongia Lopes, ${ }^{, \neq, \S}$ João A. P. Coutinho,

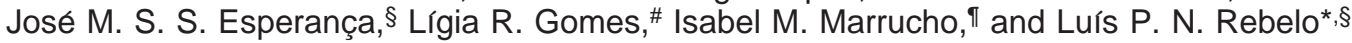 \\ Centro de Investigação em Química, Faculdade de Ciências, Universidade do Porto, R. Campo Alegre, 687, \\ 4169007 Porto, Portugal, Centro de Química Estrutural, Instituto Superior Técnico, 1049001 Lisboa, Portugal, \\ Instituto de Tecnologia Química e Biológica, Universidade Nova de Lisboa, Av. República, Ap. 127, \\ 2780901 Oeiras, Portugal, CICECO, Universidade de Aveiro, 3810193 Aveiro, Portugal, and CBFC, Faculdade \\ de Ciências da Saúde, Universidade Fernando Pessoa, R. Carlos Maia 296, 4200150 Porto, Portugal
}

Received October 17, 2006; E-mail: luis.rebelo@itqb.unl.pt; jnlopes@ist.utl.pt

In contrast to the commonly accepted view that ionic liquids are nonvolatile substances, it was recently shown by Earle et al. ${ }^{1}$ that they can be distilled under reduced pressure. This fact opens new possibilities for gas-phase processes involving ionic liquids, including their purification, high-temperature crystallization, and novel synthetic routes. It also has important theoretical implications: the nature of the gas phase has to be conclusively identified, and vapor pressure and enthalpy of vaporization data ought to be rigorously determined as they are of inestimable value for the proper validation of molecular simulation protocols as well as the anchorage of parameters for equations of state. Recently, Kabo and collaborators $^{2}$ derived vapor pressures from Knudsen-type experiments. Despite the exceptional research efforts of the past decade in the field of low-temperature molten salts, no unequivocally reliable information exists for their cohesive energy (or enthalpy of vaporization), a fact that has severely impaired both the fine testing and the development of molecular models to describe the nature and behavior of these liquid salts.

In this work, standard (0) molar (m) enthalpies of vaporization, $\Delta_{1}^{\mathrm{g}} H_{\mathrm{m}}^{0}(T)$, where $\Delta_{1}^{\mathrm{g}}=$ gas - liquid, of a series of commonly used 1-alkyl-3-methylimidazolium bis(trifluoromethyl sulfonyl)imides, $\left[\mathrm{C}_{n} \operatorname{mim}\right]\left[\mathrm{NTf}_{2}\right]$, (with $2 \leq n \leq 8$ ) were directly determined for the first time. Moreover, the results obtained in this study emphasize and constitute clear experimental support for the novel concept that these liquids are micro-biphasic systems composed of polar and nonpolar domains.

A high-precision, vacuum-vaporization drop microcalorimetric method $^{3,4}$ was used (cf. Supporting Information). This family of ionic liquids was chosen due to its thermal stability, allowing measurements to be carried out at $580 \mathrm{~K}$. All ionic liquids were synthesized and first-stage purified at the QUILL, Belfast. ${ }^{1} \mathrm{H}$ NMR, Karl-Fischer and chloride-selective electrode titrations, and mass spectra analyses showed no major impurities in the samples (cf. Supporting Information). The samples consisted of about $10 \mathrm{mg}$ of ionic liquid inserted in thin glass capillary tubes $(25-30 \mathrm{mg}$ each) sealed at one end. These were dropped at $298.15 \mathrm{~K}$ into the hot zone of the calorimeter which was kept at $577.8 \mathrm{~K}$. When the heat flow signal reached about $5 \%$ of the heat flow peak value-a sign that thermal equilibrium in the hot zone had been reachedthe liquid was completely removed from the hot zone by vaporization under vacuum. The total enthalpy change for this process is measured by time integration of the heat flow entering the calorimeter and can be subdivided in two main terms: (i) the

\footnotetext{
$\dagger$ Universidade do Porto.

¥ Instituto Superior Técnico.

\& Universidade Nova de Lisboa.

II Universidade de Aveiro.

\# Universidade Fernando Pessoa.
}

enthalpy change due to the heating of the sample in the liquid phase from the initial to the hot-zone temperature, and (ii) the enthalpy change corresponding to the vaporization of the sample at the hotzone temperature (cf. Figure 1). Both processes are endothermic, and each contribution is easily separated by fitting the missing part of the exponential decay (dotted line) after the start of the evaporation process. The $\Delta_{1}^{\mathrm{g}} H_{\mathrm{m}}^{0}(T)$ at the hot-zone temperature correspond to the area bordered by the solid and dotted lines, whereas time integration above the dotted line yields the enthalpy of heating the liquid sample from 298.15 to $577.8 \mathrm{~K}$. Table 1 reports the experimental enthalpy changes due to heating of the liquid sample from $T_{\mathrm{i}}=298.15 \mathrm{~K}$ to $T_{\mathrm{f}}=577.8 \mathrm{~K}, \Delta_{T_{\mathrm{f}}}^{T_{\mathrm{f}}} H_{\mathrm{m}}^{0}(l)$, and vaporization molar enthalpy values, $\Delta_{1}^{\mathrm{g}} H_{\mathrm{m}}^{0}(T)$, at $577.8 \mathrm{~K}$ (direct, experimental) and $298.15 \mathrm{~K}$ (indirect, calculated; see Supporting Information). The latter is useful for standardization purposes.

The experimental data were compared with simulation results. Molecular dynamics runs at 298 and $578 \mathrm{~K}$ were performed using the DL_POLY package ${ }^{5}$ and an all-atom force field based on a version of the AMBER/OPLS_AA framework extended to encompass ionic liquids of the $\left[\mathrm{C}_{n}\right.$ mim $]\left[\mathrm{NTf}_{2}\right]$ family. ${ }^{6}$ The liquid phases were modeled in boxes with 400 ions, cutoff distances of $1.2 \mathrm{~nm}$, and the usual Ewald summation technique to account for long-range interactions. More details on this type of simulation can be found elsewhere. ${ }^{7}$ Its application to compute the energetics of vaporization is novel. Since ionic liquids exhibit slow microscopic dynamics, ${ }^{8}$ equilibration runs took $1 \mathrm{~ns}$ and were followed by trajectories of $400 \mathrm{ps}$ in the $N-p-T$ ensemble at $p=0.1 \mathrm{MPa}$. The vapor phase was assumed to be formed by isolated, neutrally charged ion contact pairs in the $N-V-\mathrm{T}$ ensemble. Since the statistics are poor due to the small number of atoms, each production run took $40 \mathrm{~ns}$ and 20 such runs were used to calculate the average gas-phase properties.

The differences between the molar internal energy of the gas and liquid phases, $\Delta_{1}^{\mathrm{g}} U_{\mathrm{c}, \mathrm{m}}(T)$, as well as the contributions from van der Waals and Coulomb interactions, are given in Table 2. The $\Delta_{1}^{\mathrm{g}} H_{\mathrm{m}}^{0}(T)$ values were calculated assuming $\Delta_{1}^{\mathrm{g}} H_{\mathrm{m}}^{0}(T)=$ $\Delta_{1}^{\mathrm{g}} U_{\mathrm{c}, \mathrm{m}}(T)+R T$. Although the simulation results overestimate the experimental values by about $5-15 \%$, the predictive power of the model is very good since no data related to the energetics of these particular systems were introduced during the force field parametrization. It is worth mentioning that recently Lee and $\mathrm{Lee}^{9}$ reported estimations of internal energies of vaporization of $\left[\mathrm{C}_{n} \operatorname{mim}\right]\left[\mathrm{NTf}_{2}\right]$ at $298 \mathrm{~K}$ using the Hildebrand's solubility approach. Energetic changes upon vaporization are overestimated by $20-50 \%$.

Figure 2 shows an increase of the cohesive energy with the length of the alkyl side chain of the cation. The figure compares experimental data with those both simulated and calculated ${ }^{2}$ (Clapeyron). As for the latter, note that the evaluation of enthalpies of condensed 


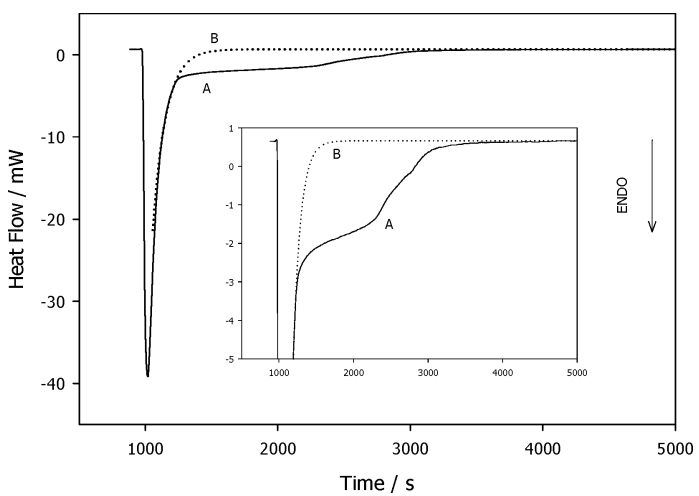

Figure 1. Typical experimental thermogram (A) and fitted exponential heat flow decay (B) obtained in a heating and vaporization experiment. The inset shows in detail the heat flow changes due to the vaporization process.

Table 1. Experimental Enthalpy Changes of $\left[\mathrm{C}_{n} \operatorname{mim}\right]\left[\mathrm{NTf}_{2}\right]^{a}$

\begin{tabular}{cccc}
\hline & & \multicolumn{2}{c}{$\Delta_{1}^{g} H_{\mathrm{m}}^{0}(T) / \mathrm{kJ} \mathrm{mol}^{-1}$} \\
\cline { 3 - 4 } ionic liquid & $\Delta_{T_{\mathrm{i}}}^{T_{\mathrm{m}}} H_{\mathrm{m}}^{0}(I) / \mathrm{kJ} \mathrm{mol}{ }^{-1}$ & & \\
$(298.15-577.8 \mathrm{~K})$ & $577.8 \mathrm{~K}$ & $298.15 \mathrm{~K}^{b}$ \\
\hline$\left[\mathrm{C}_{2}\right.$ mim $]\left[\mathrm{NTf}_{2}\right]$ & $154 \pm 5$ & $110.4 \pm 2.4$ & $136 \pm 6$ \\
{$\left[\mathrm{C}_{3}\right.$ mim $]\left[\mathrm{NTf}_{2}\right]$} & $163 \pm 3$ & $121.0 \pm 2.7$ & $147 \pm 6$ \\
{$\left[\mathrm{C}_{4}\right.$ mim $]\left[\mathrm{NTf}_{2}\right]$} & $171 \pm 5$ & $128.4 \pm 3.0$ & $155 \pm 6$ \\
{$\left[\mathrm{C}_{5}\right.$ mim $]\left[\mathrm{NTf}_{2}\right]$} & $189 \pm 6$ & $134.3 \pm 3.6$ & $162 \pm 6$ \\
{$\left[\mathrm{C}_{6}\right.$ mim $]\left[\mathrm{NTf}_{2}\right]$} & $198 \pm 6$ & $144.5 \pm 2.2$ & $173 \pm 6$ \\
{$\left[\mathrm{C}_{7}\right.$ mim $]\left[\mathrm{NTf}_{2}\right]$} & $203 \pm 10$ & $151.4 \pm 4.7$ & $180 \pm 6$ \\
{$\left[\mathrm{C}_{8}\right.$ mim $]\left[\mathrm{NTf}_{2}\right]$} & $220 \pm 6$ & $162.9 \pm 2.7$ & $192 \pm 6$ \\
\hline
\end{tabular}

${ }^{a}$ The assigned uncertainties are twice the overall standard deviation of the experimental value averages and include the calibration uncertainties. ${ }^{b}$ Estimated error $( \pm 6)$ of the calculated correction from 577.8 to $298.15 \mathrm{~K}$.

Table 2. Cohesive Energies from Simulation ${ }^{a}$

\begin{tabular}{lccccc}
\hline & \multicolumn{2}{c}{$\Delta_{1}^{g} U_{c, \mathrm{~m}}(T) / \mathrm{kJ} \mathrm{mol}^{-1}$} & & \multicolumn{2}{c}{$\Delta_{1}^{g} H_{\mathrm{m}}^{0}(T) / \mathrm{kJ} \mathrm{mol}^{-1}$} \\
\cline { 2 - 3 } ionic liquid & $577.8 \mathrm{~K}$ & $298.15 \mathrm{~K}$ & & $577.8 \mathrm{~K}$ & $298.15 \mathrm{~K}$ \\
\hline$\left[\mathrm{C}_{2}\right.$ mim $]\left[\mathrm{NTf}_{2}\right]$ & $131(70+63)$ & $157(91+62)$ & & $136 \pm 20$ & $159 \pm 10$ \\
{$\left[\mathrm{C}_{3}\right.$ mim $]\left[\mathrm{NTf}_{2}\right]$} & $143(74+77)$ & $169(95+77)$ & & $148 \pm 12$ & $172 \pm 12$ \\
{$\left[\mathrm{C}_{4}\right.$ mim $]\left[\mathrm{NTf}_{2}\right]$} & $143(76+70)$ & $171(100+70)$ & & $147 \pm 17$ & $174 \pm 11$ \\
{$\left[\mathrm{C}_{5}\right.$ mim $]\left[\mathrm{NTf}_{2}\right]$} & $150(76+69)$ & $176(104+75)$ & & $155 \pm 21$ & $179 \pm 9$ \\
{$\left[\mathrm{C}_{6}\right.$ mim $]\left[\mathrm{NTf}_{2}\right]$} & $165(85+71)$ & $182(110+74)$ & & $170 \pm 9$ & $184 \pm 7$ \\
{$\left[\mathrm{C}_{7}\right.$ mim $]\left[\mathrm{NTf}_{2}\right]$} & $158(88+72)$ & $184(114+73)$ & & $162 \pm 12$ & $186 \pm 10$ \\
{$\left[\mathrm{C}_{8}\right.$ mim $]\left[\mathrm{NTf}_{2}\right]$} & $170(92+69)$ & $198(120+69)$ & & $174 \pm 17$ & $201 \pm 6$ \\
\hline
\end{tabular}

${ }^{a}$ The values in parentheses represent the contributions of the van der Waals and Coulomb interactions, respectively. The difference of the sum to the total $\Delta_{1}^{\mathrm{g}} U_{\mathrm{c}, \mathrm{m}}(T)$ corresponds to the contribution of the internal modes.

gas-phase transitions from vapor pressure data is highly constrained by the extremely low value of this quantity for ionic liquids and the overwhelming influence of tiny amounts of impurities under these conditions. The increase of $8.9 \pm 0.6 \mathrm{~kJ} \mathrm{~mol}^{-1}$ (experimental) per each $\mathrm{CH}_{2}$ group coincides with the trend $\left(8.9 \mathrm{~kJ} \mathrm{~mol}^{-1}\right)$ observed in the solid-phase $n$-alkane family. ${ }^{10}$ Hence, this increase can be attributed to an increase in the van der Waals interactions in these highly structured liquids. However, in the case of ionic liquids, the electrostatic forces are significant and have to be accounted for. Nonetheless, somehow surprisingly, the computed Coulomb energy is noticeably constant along the homologous series. It should be noted that, if the charged parts of the cation and the anion had come further apart due to the presence of longer alkyl side chains, the electrostatic forces would have decreased along the series and the experimental $\Delta_{1}^{\mathrm{g}} H_{\mathrm{m}}^{0}(T)$ trend would not have been observed. An important piece of evidence is needed to complete a molecular interpretation: recent experimental and simulation data indicate that ionic liquids are structured fluids exhibiting segregated polar and nonpolar domains. ${ }^{7,11} \mathrm{In}$ the case of $\left[\mathrm{C}_{n} \mathrm{mim}\right]\left[\mathrm{NTf}_{2}\right]$, the polar

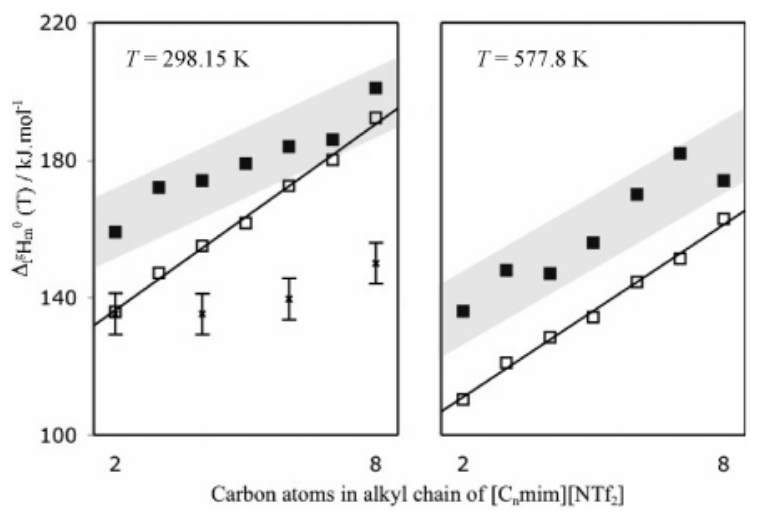

Figure 2. Molar enthalpies of vaporization of $\left[\mathrm{C}_{n} \operatorname{mim}\right]\left[\mathrm{NTf}_{2}\right]$ versus the alkyl chain length: ( $\square$ ) experimental calorimetric results and linear fit (solid

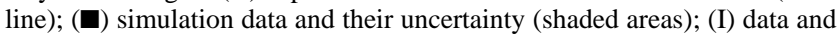
error bars estimated from Knudsen-type experiments. ${ }^{2}$ Uncertainties of calorimetric data are found in Table 1.

domains contain the anions and the imidazolium ring of the cation, whereas the nonpolar regions are formed by the alkyl side chains of the latter. In other words, ionic liquids can be seen as threedimensional polar networks permeated by nonpolar territories.

The $\Delta_{1}^{\mathrm{g}} H_{\mathrm{m}}^{0}(T)$ results presented in this work re-enforce the view of ionic liquids as structured media. As the alkyl side chains become longer, the nonpolar domains increase in size but the ionic network remains intact. Thus, while the van der Waals interactions are enhanced, the intensity of the Coulomb forces is kept invariable. This "swelling" process was observed by simulation studies ${ }^{7}$ and sheds light on the issue of the complex fluid phase behavior of ionic liquids ${ }^{12}$ and their amazing ability to dissolve very distinct solutes. The fair agreement between experimental and simulation data also seems to suggest that the vapor phase is probably composed of ion contact pairs. The current cohesive energy results can be considered as a gateway to the study of other essential energy-related properties, such as the lattice energy.

Acknowledgment. We are grateful to QUILL, led by Prof. Kenneth Seddon, for the synthesis of the ILs.

Supporting Information Available: Experimental details. This material is available free of charge via the Internet at http://pubs.acs.org.

\section{References}

(1) (a) Earle, M. J.; Esperança, J. M. S. S.; Gilea, M. A.; Canongia Lopes, J. N.; Rebelo, L. P. N.; Magee, J. W.; Seddon, K. R.; Widegren, J. A. Nature 2006, 439, 831-834. (b) Rebelo, L. P. N.; Canongia Lopes, J. N.; Esperança, J. M. S. S.; Filipe, E. J. Phys. Chem. B 2005, 109, 60406043. (c) Wasserscheid, P. Nature 2006, 439, 797.

(2) Zaitsau, D. H.; Kabo, G. J.; Strechan, A. A.; Paulechka, Y. U.; Tschersich, A.; Verevkin, S. P.; Heintz, A. J. Phys. Chem. A 2006, 110, 7303-7306.

(3) Adedeji, F. A.; Brown, D. L. S.; Connor, J. A.; Leung, M.; Paz-Andrade, M. I.; Skinner, H. A. J. Organomet. Chem. 1975, 97, 221-228.

(4) Santos, L. M. N. B. F.; Schröder, B.; Fernandes, O. O. P.; Ribeiro da Silva, M. A. V. Thermochim. Acta 2004, 415, 15-20.

(5) Smith, W.; Forester, T. R. DL-POLY package 2.12; CCLRC: Daresbury Laboratory, Warrington, UK, 1999.

(6) (a) Canongia Lopes, J. N.; Deschamps, J.; Pádua, A. A. H. J. Phys. Chem. B 2004, 108, 2038-2047. (b) Canongia Lopes, J. N.; Pádua, A. A. H. J. Phys. Chem. B 2004, 108, 16893-16898.

(7) (a) Pádua, A. A. H.; Canongia Lopes, J. N. J. Phys. Chem. B 2006, 110 3330-3335. (b) Canongia Lopes, J. N.; Costa Gomes, M. F.; Pádua, A A. H. J. Phys. Chem. B 2006, 110, 16816-16818.

(8) Cadena, C.; Zhao, Q.; Snurr, R. Q.; Maginn, E. J. J. Phys. Chem. B 2006 $110,2821-2832$.

(9) Lee, S. H.; Lee, S. B. Chem. Commun. 2005, 3469-3471

(10) (a) Matulis, D. Biophys. Chem. 2001, 93, 67-82. (b) Chickos, J. S.; Hanshaw W. J. Chem. Eng. Data 2004, 49, 620-630.

(11) Wang, Y.; Voth, G. A. J. Am. Chem. Soc. 2005, 127, 12192-12193.

(12) Lachwa, J.; Szydlowski, J.; Najdanovic-Visak, V.; Rebelo, L. P. N.; Seddon, K. R.; Nunes da Ponte, M.; Esperança, J. M. S. S.; Guedes, H. J. R. J. Am. Chem. Soc. 2005, 127, 6542-6543.

JA067427B 